\title{
Particles and the Universe
}

\section{George Lazarides*}

School of Electrical and Computer Engineering, Faculty of Engineering, Aristotle University of Thessaloniki, Thessaloniki 54124, Greece

E-mail: lazaridedeng.auth.gr

The early stages of the universe evolution are discussed according to the hot big bang model and the grand unified theories. The shortcomings of big bang are summarized and their resolution by inflationary cosmology is sketched. Cosmological inflation, the subsequent oscillation and decay of the inflaton field, and the resulting reheating of the universe are studied in some detail. The density perturbations produced by inflation and the temperature fluctuations of the cosmic microwave background radiation are introduced. Baryogenesis via non-thermal leptogenesis is analyzed and dark energy and matter in the universe are presented. Quantum gravity and string theory are very briefly introduced. The problem of initial conditions for inflation is discussed in the light of string theory and the possibly detectable primordial gravity waves from inflation are mentioned.

Corfu Summer Institute 2019 "School and Workshops on Elementary Particle Physics and Gravity" (CORFU2019)

31 August - 25 September 2019

Corfu, Greece

${ }^{*}$ Speaker. 


\section{Introduction}

The observed Hubble expansion of the universe, the discovery of the cosmic microwave background radiation (CMBR) and the success of nucleosynthesis (see e.g. Ref. [1]) in reproducing the observed abundance of light elements in the universe had established the standard big bang (SBB) cosmological model (for a textbook treatment, see e.g. Ref. [2]). This model together with the grand unified theories (GUTs) [3] of strong, weak, and electromagnetic interactions provides the scientific framework for studying the early stages of the universe.

Despite its great successes, the SBB model had some shortcomings. One of them is the socalled horizon problem. The CMBR received now has been emitted from regions of the universe which, according to the SBB model, had never communicated before sending this radiation to us. The question then arises how come the temperature of this radiation from these regions is so finely tuned as the measurements of the COBE [4], the WMAP [5], and the Planck [6] satellites show. Another important puzzle is the so-called flatness problem. It is a fact [6] that the present universe appears to be very flat. This means that, in its early stages, the universe must have been flat with a great accuracy, which requires an extreme fine tuning of its initial conditions. Also, combined with GUTs which predict the existence of superheavy magnetic monopoles [7], the SBB model leads [8] to a catastrophic overproduction of these monopoles. Finally, the model has no explanation for the small density perturbations required for explaining the structure formation in the universe (for a pedagogical discussion, see e.g. Ref. [9]) and the generation of the observed $[4,5,6]$ temperature fluctuations in the CMBR.

Cosmological inflation [10] offers a solution to all these problems of the SBB model (for a textbook introductions or reviews on inflation, see e.g. Ref. [11]). The idea is that, in the early universe, a real scalar field (the inflaton) was displaced from its vacuum value. If the potential of this field is quite flat, the roll-over of the field towards the vacuum can be very slow for a period of time during which the energy density of the universe is dominated by the almost constant potential energy density of the inflaton. Consequently, the universe undergoes a period of quasi-exponential expansion, which can readily solve the horizon and flatness problems by stretching the distance over which causal contact is established and reducing any pre-existing curvature in the universe. It can also adequately dilute the GUT magnetic monopoles and provide us with the primordial density perturbations which are needed for explaining the large scale structure in the universe [9] and the temperature fluctuations observed in the CMBR. Inflation can occur during the GUT phase transition at which the GUT gauge symmetry breaks by the vacuum expectation value (VEV) of a Higgs field, which also plays the role of the inflaton.

After the end of inflation, the inflaton starts performing damped oscillations about the vacuum and decays into light particles. The resulting radiation energy density eventually dominates over the field energy density and the universe returns to a normal big bang type evolution. The temperature at which this occurs is historically called reheat temperature although there is neither supercooling nor reheating of the universe [12] (see also Ref. [13]).

An acceptable inflationary scenario must necessarily be followed by a successful reheating process which, in the case of supersymmetry, must satisfy the gravitino constraint [14] on the reheat temperature, $T_{\mathrm{r}} \lesssim 10^{9} \mathrm{GeV}$. This process must also generate the observed baryon asymmetry of the universe. In inflationary models, it is generally preferable to generate the baryon asymmetry 
by non-thermal [15] leptogenesis [16], i.e. by first producing a primordial lepton asymmetry from the decay products of the inflaton. This asymmetry is then partly converted into baryon asymmetry by the non-perturbative electroweak sphaleron effects [17, 18]. Actually, in many specific models, this is the only way to generate the baryon asymmetry of the universe since the inflaton decays into right handed neutrinos. The subsequent decay of these fields into a lepton (antilepton) and an electroweak Higgs field can only produce a lepton asymmetry.

Recent measurements [6] confirmed the prediction of inflation that the present universe is flat. They also reveal that matter constitutes only $27 \%$ of the universe. The rest $73 \%$ of the universe is in the form of dark energy, i.e. in a form close to a cosmological constant. This means that this energy is basically not diluted by the expansion of the universe exactly as the energy driving inflation. On the other hand, the baryonic (visible) matter constitutes only $4.85 \%$ of the universe as nucleosynthesis [1] and the Planck satellite [6] have shown. Consequently, about $22 \%$ of the universe is made of some form of dark matter. The most promising candidate for dark matter [19] is the lightest supersymmetric particle (LSP) since it is protected by a discrete $Z_{2}$ R-parity symmetry from decaying into lighter particles. The LSPs can annihilate in pairs or coannihilate with the nextto-LSPs. Their relic density can be reduced to the observed value of dark matter abundance mainly by resonant pair annihilation [20] or coannihilation [21] with next-to-LSPs which happened to be almost degenerate with them. Other possible dark matter candidates include the axion [22] and superheavy [23] or intermediate scale mass [24, 25] fermions.

At cosmic times greater than the Planck time $t_{\mathrm{P}} \sim 10^{-43} \mathrm{sec}$, gravity is adequately described by the classical theory of general relativity. However, for smaller cosmic times, quantum fluctuations of gravity are present. Therefore, it is desirable to have a quantum theory of gravity and if possible unify gravity with the other three interactions, which are described by relativistic quantum field theory, so as to obtain the theory of everything. However, in contrast with the other three interactions, the quantum field theory for gravity is not renormalizable. This means that the infinities appearing in the various calculations and originating from the point-like character of particles cannot be systematically gathered in a finite number of parameters. Consequently, the theory is lacking predictability. The theory of (super)strings [26] was proposed in order to cure this difficulty. The idea is that the fundamental objects are not point-like particles but one dimensional strings of size $\ell_{\mathrm{P}} \sim 10^{-33} \mathrm{~cm}$. Their various vibrational modes, one of which is the graviton, appear as particles with different quantum numbers. The main disadvantage of string theory is that it admits a huge number of solutions $\left(\sim 10^{500}\right)$, but none of them reproduces exactly our universe. It predicts that there exist ten spacetime dimensions (or eleven in the case of M theory). Six of them are compactified to form a 6-dimensional compact space of size $\ell_{\mathrm{P}}$, while the other four dimensions remain open and are the usual spacetime dimensions. The geometric structure of the compactified dimensions determines many of the phenomena in the 4-dimensional spacetime.

One problem we can address in the light of string theory is the problem of initial conditions [27] for inflation which takes place at the GUT transition at $t \sim 10^{-37} \mathrm{sec} \gg t_{\mathrm{P}}$. At the onset of inflation, one needs a large homogeneous region around the GUT phase transition. However, this region consists of many smaller homogeneous regions originating from the Planck era, where causal communication is restricted to distances of the order of the Planck length. The resolution of this problem requires a first stage of inflation near or before the big bang, which provides the necessary homogenization for the onset of conventional inflation. Such a primordial inflation can 
take place in the pre-big-bang period. During the motion of the universe towards the initial singularity, we have conditions of very high curvature and the extra dimensions contract and compactify. This causes inflation in the four open dimensions. Another application of quantum gravity could be the explanation of the origin of primordial gravity waves if such waves are detected in the future. The Planck satellite measurements [6] imply an upper bound on the tensor-to-scalar ratio $r \lesssim 0.06$. Therefore, one cannot exclude that future experiments may detect primordial gravity waves originating from inflation. Quantum gravity may then be required to understand the origin of these waves.

In Sec. 2, we summarize the salient features of SBB cosmology, while in Sec. 3 we sketch the series of phase transitions the universe underwent in accordance with GUTs. The shortcomings of big bang and their resolution by inflation are discussed in Secs. 4 and 5, respectively. Sec. 6 is devoted to the detailed discussion of inflation, reheating, and density and temperature fluctuations. Baryogenesis via leptogenesis is the subject of Sec. 7. In Secs. 8 and 9, we review the composition of the universe, while in Secs. 10 and 11 we briefly refer to quantum gravity and string theory with some possible applications in cosmology. Finally, in Sec. 12 we summarize our conclusions.

\section{Big Bang Cosmology}

We start with an introduction to the SBB model [2]. For cosmic times $t \gtrsim t_{\mathrm{P}} \equiv m_{\mathrm{P}}^{-1} \sim 10^{-43} \mathrm{sec}$ $\left(m_{\mathrm{P}} \simeq 2.44 \times 10^{18} \mathrm{GeV}\right.$ is the reduced Planck scale) after the big bang, the quantum fluctuations of gravity cease to exist and gravitation can be described by the classical theory of general relativity. Strong, weak, and electromagnetic interactions, however, are described by gauge theories which are relativistic quantum field theories.

An important starting point is the so-called cosmological principle, which states that, at large scales, the universe is homogeneous and isotropic. The strongest evidence for this is the observed $[4,5,6]$ isotropy of the CMBR. The spacetime metric then takes the Robertson-Walker form

$$
d s^{2}=-d t^{2}+a^{2}(t)\left[\frac{d r^{2}}{1-k r^{2}}+r^{2}\left(d \vartheta^{2}+\sin ^{2} \vartheta d \varphi^{2}\right)\right],
$$

where $r, \varphi$, and $\vartheta$ are comoving polar coordinates, remaining fixed for objects that just follow the cosmological expansion. The parameter $k$ is the scalar curvature of the 3 -space and $k=0,>0$, or $<0$ corresponds to flat, closed, or open universe. The dimensionless parameter $a(t)$ is the scale factor of the universe. It is normalized so that $a_{0} \equiv a\left(t_{0}\right)=1$, where $t_{0}$ is the present cosmic time.

The instantaneous radial physical distance is given by

$$
R=a(t) \int_{0}^{r} \frac{d r}{\left(1-k r^{2}\right)^{\frac{1}{2}}} .
$$

For flat universe $(k=0), \bar{R}=a(t) \bar{r}(\bar{r}$ is a comoving and $\bar{R}$ a physical radial vector in 3-space) and the velocity of a comoving object is

$$
\bar{V}=\frac{\dot{a}}{a} \bar{R} \equiv H(t) \bar{R},
$$

where the overdot denotes derivation with respect to $t$ and $H(t)$ is the Hubble parameter. This equation is the well-known Hubble law asserting that all objects run away from each other with velocities proportional to their distances. This law is the first success of SBB cosmology. 
Energy-momentum conservation yields the continuity equation

$$
\frac{d \rho}{d t}=-3 H(t)(\rho+p)
$$

where $\rho$ and $p$ are the energy density and pressure in the universe. The first term in the right-hand side (RHS) of this equation describes the dilution of the energy due to the Hubble expansion and the second term the work done by pressure. Einstein's equations for the Robertson-Walker metric take the form of the Friedmann equation

$$
H^{2} \equiv\left(\frac{\dot{a}(t)}{a(t)}\right)^{2}=\frac{8 \pi G}{3} \rho-\frac{k}{a^{2}},
$$

where $G$ is Newton's gravitational constant. Averaging the pressure $p$, we write $\rho+p=\gamma \rho$ and Eq. (2.4) gives $\rho \propto a^{-3 \gamma}$. From Eq. (2.5) with $k=0$, we then get $a(t) \propto t^{2 / 3 \gamma}$. For a universe dominated by pressureless matter, $\gamma=1$ and, thus, $\rho \propto a^{-3}$ and $a(t)=\left(t / t_{0}\right)^{2 / 3}$. This is interpreted as mere dilution of a fixed number of particles in a comoving volume due to the Hubble expansion. For a radiation dominated universe, $p=\rho / 3$ and, thus, $\gamma=4 / 3$, which gives $\rho \propto a^{-4}$ and $a(t)=$ $\left(t / t_{0}\right)^{1 / 2}$. The extra factor of $a(t)$ in the energy dilution is due to the red-shifting of all wave lengths by the Hubble expansion.

The early universe is radiation dominated and its energy density is given by

$$
\rho=\frac{\pi^{2}}{30} g_{*} T^{4},
$$

where $T$ is the cosmic temperature and $g_{*}=N_{b}+(7 / 8) N_{f}$ is the effective number of massless degrees of freedom with $N_{b(f)}$ being the number of massless bosonic (fermionic) degrees of freedom. The temperature-time relation during radiation dominance is derived from Eq. (2.5) (with $k=0$ ):

$$
T^{2}=\frac{3 \sqrt{5} m_{\mathrm{P}}}{\sqrt{2 g_{*}} \pi t}, \quad m_{\mathrm{P}} \equiv(8 \pi G)^{-\frac{1}{2}}=\text { the reduced Planck mass. }
$$

Classically, the expansion starts at $t=0$ with $T=\infty$ and $a=0$. This initial singularity is, however, not physical since general relativity fails for $t \lesssim t_{\mathrm{P}}$. The only meaningful statement is that the universe, after a yet unknown initial stage, emerges at $t \sim t_{\mathrm{P}}$ with $T \sim m_{\mathrm{P}}$.

An important notion is the notion of particle horizon $d_{H}(t)$, which is the instantaneous distance at $t$ traveled by light since the beginning of time $(t=0)$. From Eq. (2.1), we find that

$$
d_{H}(t)=a(t) \int_{0}^{t} \frac{d t^{\prime}}{a\left(t^{\prime}\right)}
$$

which is finite and coincides with the size of the universe already seen at time $t$ or, equivalently, with the distance over which causal contact has been established at $t$. For matter (radiation) dominated universe, we have $d_{H}(t)=2 H^{-1}(t)=3 t\left(d_{H}(t)=H^{-1}(t)=2 t\right)$. After the Planck satellite measurements [6], the present age of our universe is estimated to be $t_{0} \simeq(13.801 \pm 0.024) \times 10^{9}$ years, the present value of the Hubble parameter $H_{0}=100 \mathrm{~h} \mathrm{~km} \mathrm{sec}^{-1} \mathrm{Mpc}^{-1}$ with $h \simeq 0.674 \pm 0.005$, and the present critical density corresponding to a flat universe $\rho_{\mathrm{c}}=3 H_{0}^{2} / 8 \pi G \simeq 0.86 \times 10^{-29} \mathrm{gm} / \mathrm{cm}^{3}$. The fraction of the actual to the critical density is $\Omega \equiv \rho / \rho_{\mathrm{c}} \simeq 1 \pm 0.01$, which means that our present universe is very flat. 


\section{Phase Transitions in the Universe}

GUTs together with the SBB model predict that, as the universe expands and cools after the big bang, it undergoes [28] a series of phase transitions during which the GUT gauge symmetry is gradually reduced and several important phenomena take place. For definiteness, we consider here a simple non-supersymmetric $S U(5)$ GUT model, but the discussion can be readily extended to include other gauge groups such as $E_{6}, S O(10), S U(4)_{\mathrm{c}} \times S U(2)_{\mathrm{L}} \times S U(2)_{\mathrm{R}}$, and $S U(3)^{3}$ [29] with or without supersymmetry. At a scale $M_{\mathrm{G}} \sim 10^{16} \mathrm{GeV}$ (the GUT mass scale), $S U(5)$ breaks to the standard model gauge group $G_{\mathrm{SM}}=S U(3)_{\mathrm{c}} \times S U(2)_{\mathrm{L}} \times U(1)_{Y}$ by the VEV of an appropriate Higgs field $\phi$. Subsequently, $G_{\mathrm{SM}}$ is broken to $S U(3)_{\mathrm{c}} \times U(1)_{\mathrm{em}}$ at the electroweak scale $M_{\mathrm{W}}\left(\mathrm{SU}(3)_{\mathrm{c}}\right.$ and $\mathrm{U}(1)_{\mathrm{em}}$ are, respectively, the gauge groups of strong and electromagnetic interactions).

Initially, $S U(5)$ was unbroken and the universe was filled with a hot soup of massless particles including photons, quarks, leptons, gluons, weak gauge bosons $W^{ \pm}, Z^{0}$, GUT gauge bosons $X$, $Y$, and several Higgs bosons. At $t \sim 10^{-37} \sec \left(T \sim 10^{16} \mathrm{GeV}\right), S U(5)$ broke down to $G_{\mathrm{SM}}$ and the $X, Y$ bosons and some Higgs bosons acquired masses $\sim M_{\mathrm{G}}$. Their out-of-equilibrium decay could, in principle, generate $[17,30]$ the observed baryon asymmetry of the universe, i.e. an excess of baryons over antibaryons. Important ingredients are the violation of baryon number, which is inherent in GUTs, and C and CP violation. This is the second (potential) success of the SBB model.

Moreover, at the GUT phase transition, topologically stable extended objects [31] such as magnetic monopoles [7], cosmic strings [32], or domain walls [33] can also be generated. Monopoles, which exist in most GUTs, can lead into problems [8] which are, however, avoided by inflation $[10,11]$ (see below). This is a period of exponentially fast expansion of the universe which can occur during the GUT phase transition and can totally remove the monopoles from the scene. Alternatively, a more moderate inflation such as thermal inflation [34], associated with a phase transition occurring at a temperature of the order of the electroweak scale, can dilute them to an acceptable, but possibly measurable level. Cosmic strings from GUTs [35], on the other hand, can generate gravity waves [36], which will be possibly measurable [24, 37, 38] by future experiments. Finally, domain walls are [33] catastrophic and GUTs should be constructed so that they avoid them (see e.g. Ref. [39]) or inflation should extinguish them. Note that, in some cases, more complex extended objects such as walls bounded by strings [40] or strings connecting monopoles [41] can be temporarily produced.

At $t \sim 10^{-10} \sec (T \sim 100 \mathrm{GeV})$, the electroweak phase transition takes place and $G_{\mathrm{SM}}$ breaks to $\mathrm{SU}(3)_{\mathrm{c}} \times \mathrm{U}(1)_{\mathrm{em}}$. The electroweak Higgs field as well as the weak gauge bosons $W^{ \pm}, Z^{0}$ acquire masses $\sim M_{\mathrm{W}}$. Subsequently, at $t \sim 10^{-4} \sec (T \sim 1 \mathrm{GeV})$, color is confined and the quarks come together to form hadrons. The direct involvement of particle physics ends here. We will, however, sketch some of the subsequent cosmological events since they provide crucial information on the early universe, where their origin lies.

At $t \simeq 180 \mathrm{sec}(T \simeq 1 \mathrm{MeV})$, nucleosynthesis takes place, i.e. protons and neutrons form nuclei. The abundance of light elements (D, ${ }^{3} \mathrm{He},{ }^{4} \mathrm{He},{ }^{6} \mathrm{Li}$, and ${ }^{7} \mathrm{Li}$ ) depends (see e.g. Ref. [42]) crucially on the number of light particles (with mass $\lesssim 1 \mathrm{MeV}$ ), i.e. the number $N_{V}$ of light neutrinos and the baryon abundance $\Omega_{\mathrm{B}} h^{2}\left(\Omega_{\mathrm{B}}=\rho_{\mathrm{B}} / \rho_{\mathrm{c}}\right.$ with $\rho_{\mathrm{B}}$ being the baryon energy density). Agreement with observations [43] can be achieved for $N_{v}=3$ and $\Omega_{\mathrm{B}} h^{2} \simeq 0.02$. This is the third success of SBB cosmology. Much later, at the so-called equidensity time $t_{\text {eq }} \simeq 4.7 \times 10^{4}$ years, 
matter dominates over radiation. At $t \simeq 200,000 h^{-1}$ years $(T \simeq 3,000 \mathrm{~K})$, the decoupling of matter and radiation and the recombination of atoms occur. After this, radiation evolves independently and is detected today as CMBR with temperature $T_{0} \simeq 2.73 \mathrm{~K}$. The existence of the CMBR is the fourth success of SBB. Finally, structure formation [9] starts at $t \simeq 2 \times 10^{8}$ years.

\section{Shortcomings of SBB cosmology}

The SBB model has been very successful in explaining, among other things, the Hubble expansion of the universe, the existence of the CMBR, and the abundance of the light elements formed during nucleosynthesis. Moreover, combined with GUTs provides the basis for generating the baryon asymmetry of the universe, i.e. the slight excess of baryons over antibaryons, so that after baryon-antibaryon annihilation there are leftover baryons out of which the visible part of the universe is made. Despite its successes, this model had a number of very puzzling shortcomings which we will now summarize:

1. The horizon problem: The CMBR was emitted at the decoupling of matter and radiation when $T_{\mathrm{d}} \simeq 3,000 \mathrm{~K}$. The decoupling time $t_{\mathrm{d}}$ is estimated from

$$
\frac{T_{0}}{T_{\mathrm{d}}}=\frac{a\left(t_{\mathrm{d}}\right)}{a\left(t_{0}\right)}=\left(\frac{t_{\mathrm{d}}}{t_{0}}\right)^{\frac{2}{3}} \simeq \frac{2.73 \mathrm{~K}}{3,000 \mathrm{~K}}
$$

and turns out to be $t_{\mathrm{d}} \simeq 200,000 \mathrm{~h}^{-1}$ years. The distance over which the CMBR has traveled since its emission is

$$
a\left(t_{0}\right) \int_{t_{\mathrm{d}}}^{t_{0}} \frac{d t^{\prime}}{a\left(t^{\prime}\right)}=3 t_{0}\left[1-\left(\frac{t_{\mathrm{d}}}{t_{0}}\right)^{\frac{2}{3}}\right] \simeq 3 t_{0} \simeq 6,000 \mathrm{~h}^{-1} \mathrm{Mpc},
$$

which practically coincides with $d_{H}\left(t_{0}\right)$. A sphere of radius $d_{H}\left(t_{0}\right)$ around us is called the last scattering surface since the CMBR has been emitted from it. The particle horizon at decoupling $3 t_{\mathrm{d}} \simeq 0.168 h^{-1} \mathrm{Mpc}$, expanded until now to become $0.168 h^{-1}\left(a\left(t_{0}\right) / a\left(t_{\mathrm{d}}\right)\right) \mathrm{Mpc} \simeq$ $184 h^{-1}$ Mpc. The angle subtended by this decoupling horizon now is $\vartheta_{\mathrm{d}} \simeq 184 / 6,000 \simeq$ 0.03 rads. Thus, the sky splits into $4 \pi /(0.03)^{2} \simeq 14,000$ patches which never communicated causally before emitting the CMBR. The puzzle then is how can the temperature of the black body radiation from these patches be so finely tuned as the measurements of the Planck satellite [6] require.

2. Flatness Problem: The present energy density of the universe has been observed [6] to be very close to its critical value corresponding to a flat universe $\left(\Omega_{0}=1 \pm 0.001\right)$. From Eq. (2.5), we obtain $\left(\rho-\rho_{\mathrm{c}}\right) / \rho_{\mathrm{c}}=3\left(8 \pi G \rho_{\mathrm{c}}\right)^{-1}\left(k / a^{2}\right) \propto a$ for a matter dominated universe. Thus, in the early universe, $\left|\left(\rho-\rho_{\mathrm{c}}\right) / \rho_{\mathrm{c}}\right| \ll 1$ and the question is why the initial energy density of the universe was so finely tuned to its critical value.

3. Magnetic Monopole Problem: This problem arises only if we combine SBB with GUTs [3] which predict the existence of magnetic monopoles [7]. These monopoles are produced during the GUT phase transition, where the Higgs field $\phi$ responsible for the breaking of the 
GUT gauge symmetry $G$ acquires its VEV. They are localized deviations from the vacuum with radius $\sim M_{\mathrm{G}}^{-1}$ and mass $m_{\mathrm{M}} \sim M_{\mathrm{G}} / \alpha_{\mathrm{G}}\left(\alpha_{\mathrm{G}}=g_{\mathrm{G}}^{2} / 4 \pi\right.$, where $g_{\mathrm{G}}$ is the GUT gauge coupling constant). The value of $\phi$ on a sphere $S^{2}$ of radius $\gg M_{\mathrm{G}}^{-1}$ around the monopole lies in the vacuum manifold $G / G_{\mathrm{SM}}$ and we, thus, obtain a mapping: $S^{2} \rightarrow G / G_{\mathrm{SM}}$. If this mapping is homotopically non-trivial, the monopole is topologically stable. The initial relative monopole number density must satisfy the causality bound [44] $r_{\mathrm{M} \text {,in }} \equiv\left(n_{\mathrm{M}} / T^{3}\right)_{\text {in }} \gtrsim$ $10^{-10}$ ( $n_{\mathrm{M}}$ is the monopole number density), which comes from the requirement that, at monopole production, $\phi$ cannot be correlated at distances bigger than the particle horizon. The subsequent evolution of monopoles is studied in Ref. [8]. The result is that, if $r_{\mathrm{M} \text {,in }} \gtrsim$ $10^{-9}\left(\lesssim 10^{-9}\right)$, the final relative monopole number density $r_{\mathrm{M} \text {,fin }} \sim 10^{-9}\left(\sim r_{\mathrm{M}, \mathrm{in}}\right)$. This combined with the causality bound yields $r_{\mathrm{M}, \mathrm{fin}} \gtrsim 10^{-10}$. However, the requirement that monopoles do not dominate the energy density of the universe at nucleosynthesis gives

$$
r_{\mathrm{M}}(T \simeq 1 \mathrm{MeV}) \lesssim 10^{-19}
$$

and we obtain a clear discrepancy of about nine orders of magnitude.

4. Density Perturbations: For structure formation [9] in the universe, we need a primordial density perturbation $\delta \rho / \rho$ at all length scales with a nearly flat spectrum [45]. We also need an explanation of the temperature fluctuations of the CMBR measured by the Planck satellite [6] at angles $\vartheta \gtrsim \vartheta_{\mathrm{d}} \simeq 2^{\circ}$, which violate causality. The SBB model cannot provide the required perturbations.

\section{Inflation}

All these four puzzles are solved by inflation [10,11], which is a period of exponential expansion in the early universe. Consider a real scalar field $\phi$ (the inflaton) with a (symmetric) potential $V(\phi)$ which is quite flat near $\phi=0$ and has minima at $\phi= \pm\langle\phi\rangle$ with $V( \pm\langle\phi\rangle)=0$. At high $T$ 's, the potential acquires temperature corrections, which make $\phi=0$ the absolute minimum. As the temperature drops, the effective potential tends to the zero-temperature potential, but a small barrier separating the local minimum at $\phi=0$ and the vacua at $\phi= \pm\langle\phi\rangle$ remains. At some point, $\phi$ tunnels out to $\phi_{1} \ll\langle\phi\rangle$ and a bubble with $\phi=\phi_{1}$ is created in the universe. The field then rolls over to the minimum of $V(\phi)$ very slowly (due to the flatness of the potential $V(\phi)$ ) with the energy density $\rho \simeq V(\phi=0) \equiv V_{0}$ remaining practically constant for quite some time. The energy-momentum tensor during the slow roll-over of the inflaton becomes $T_{\mu}{ }^{v} \simeq-V_{0} \delta_{\mu}{ }^{v}$ yielding $\rho \simeq-p \simeq V_{0}$. So, the pressure is practically opposite to the energy density, which remains almost constant in accord with Eq. (2.4). The scale factor $a(t)$ grows (see below) and the curvature term $k / a^{2}$ in Eq. (2.5) diminishes. We thus obtain $H^{2} \simeq 8 \pi G V_{0} / 3=$ constant, which gives $a(t) \propto e^{H t}$. Therefore, the bubble expands exponentially for some time and $a(t)$ grows by a factor

$$
\frac{a\left(t_{\mathrm{f}}\right)}{a\left(t_{\mathrm{i}}\right)}=\exp H\left(t_{\mathrm{f}}-t_{\mathrm{i}}\right) \equiv \exp H \tau
$$

between an initial $\left(t_{\mathrm{i}}\right)$ and a final $\left(t_{\mathrm{f}}\right)$ time.

It is almost obvious that, with an adequate number of $e$-foldings $N \equiv H \tau$, inflation automatically resolves the first three puzzles of SBB: 
1. Resolution of the Horizon Problem: The particle horizon during inflation

$$
d_{H}(t)=e^{H t} \int_{t_{\mathrm{i}}}^{t} \frac{d t^{\prime}}{e^{H t^{\prime}}} \simeq H^{-1} \exp H\left(t-t_{\mathrm{i}}\right)
$$

for $t-t_{\mathrm{i}} \gg H^{-1}$, grows as fast as $a(t)$. At $t_{\mathrm{f}}$ where inflation ends, $d_{H}\left(t_{\mathrm{f}}\right) \simeq H^{-1} \exp H \tau$ and $\phi$ starts oscillating about the vacuum. It then decays and reheats [12] the universe at a temperature $T_{\mathrm{r}} \sim 10^{9} \mathrm{GeV}$ [14] after which normal big bang cosmology is recovered. The particle horizon at the end of inflation $d_{H}\left(t_{\mathrm{f}}\right)$ is stretched during the $\phi$-oscillations by a factor $\sim 10^{9}$ and between $T_{\mathrm{r}}$ and the present time by a factor $T_{\mathrm{r}} / T_{0}$. So, it finally becomes equal to $H^{-1} e^{H \tau} 10^{9}\left(T_{\mathrm{r}} / T_{0}\right)$, which must exceed $2 H_{0}^{-1}$ if the horizon problem is to be solved. This readily holds for $V_{0} \sim M_{\mathrm{G}}^{4}, M_{\mathrm{G}} \sim 10^{16} \mathrm{GeV}$, and $N \gtrsim 55$.

2. Resolution of the Flatness Problem: The curvature term of the Friedmann equation, at present, is

$$
\left(\frac{k}{a^{2}}\right)_{0} \simeq\left(\frac{k}{a^{2}}\right)_{\mathrm{bi}} e^{-2 H \tau} 10^{-18}\left(\frac{10^{-13} \mathrm{GeV}}{10^{9} \mathrm{GeV}}\right)^{2},
$$

where the factors in the RHS are the curvature term before inflation and its growth factors during inflation, $\phi$-oscillations, and after reheating. Assuming $\left(k / a^{2}\right)_{\mathrm{bi}} \sim H^{2}$, we obtain $\Omega_{0}-1=k / a_{0}^{2} H_{0}^{2} \sim 10^{48} e^{-2 H \tau} \ll 1$ for $H \tau \gg 55$. Strong inflation implies that the present universe is flat with a great accuracy.

3. Resolution of the Monopole Problem: For $N \gtrsim 55$, the magnetic monopoles are diluted by at least 70 orders of magnitude and become irrelevant. Also, since $T_{\mathrm{r}} \ll m_{\mathrm{M}}$, there is no magnetic monopole production after reheating. For models leading to a possibly measurable magnetic monopole density, see e.g. Refs. [25, 34, 38, 46].

4. Generation of density perturbations: Inflation transforms the quantum fluctuations of the almost massless inflaton field into classical metric perturbations, which in turn generate tiny primordial density perturbations $\delta \rho / \rho \simeq 5.6 \times 10^{-5}$. These perturbations grow in the late universe to become non-linear and lead to the formation of structure (galaxies, filaments, and great voids) via gravitational collapse of matter. They also generate the temperature fluctuation $\delta T / T \simeq 6 \times 10^{-6}$ in the CMBR measured by the COBE, WMAP, and Planck satellites. The predictions of inflation can fully agree with all experimental findings.

\section{Details of Inflation}

The Hubble parameter during inflation is slowly varying with the value of $\phi$ :

$$
H^{2}(\phi)=\frac{8 \pi G}{3} V(\phi) .
$$

The evolution equation for $\phi$ is

$$
\ddot{\phi}+3 H \dot{\phi}+\Gamma_{\phi} \dot{\phi}+V^{\prime}(\phi)=0,
$$

where the dot and prime denote derivation with respect to the cosmic time and $\phi$, respectively, and $\Gamma_{\phi}$ is the decay width [47] of the inflaton to light particles, which is assumed to be weak $\left(\Gamma_{\phi} \ll H\right)$ 
[48]. Inflation is by definition the situation where the friction term $3 H \dot{\phi}$ dominates and Eq. (6.2) reduces to the inflationary equation [49]

$$
3 H \dot{\phi}=-V^{\prime}(\phi) .
$$

The conditions for this equation to hold (slow roll conditions) are:

$$
\varepsilon,|\eta| \leq 1 \text { with } \varepsilon \equiv \frac{1}{2} m_{\mathrm{P}}^{2}\left(\frac{V^{\prime}(\phi)}{V(\phi)}\right)^{2}, \eta \equiv m_{\mathrm{P}}^{2} \frac{V^{\prime \prime}(\phi)}{V(\phi)} .
$$

The end of the slow roll-over occurs when either of these inequalities is saturated. If $\phi_{\mathrm{f}}$ is the value of $\phi$ at the end of inflation, then $t_{\mathrm{f}} \sim H^{-1}\left(\phi_{\mathrm{f}}\right)$.

The number of $e$-foldings during inflation from $t_{\mathrm{i}}\left(\right.$ or $\left.\phi_{\mathrm{i}}\right)$ to $t_{\mathrm{f}}\left(\right.$ or $\left.\phi_{\mathrm{f}}\right)$ is

$$
N\left(\phi_{\mathrm{i}} \rightarrow \phi_{\mathrm{f}}\right) \equiv \ln \left(\frac{a\left(t_{\mathrm{f}}\right)}{a\left(t_{\mathrm{i}}\right)}\right)=\int_{t_{\mathrm{i}}}^{t_{\mathrm{f}}} H d t=\int_{\phi_{\mathrm{i}}}^{\phi_{\mathrm{f}}} \frac{H(\phi)}{\dot{\phi}} d \phi=-\int_{\phi_{\mathrm{i}}}^{\phi_{\mathrm{f}}} \frac{3 H^{2}(\phi) d \phi}{V^{\prime}(\phi)},
$$

where Eqs. (5.1) and (6.3) were used.

After the end of inflation at $t_{\mathrm{f}}, \phi$ starts performing coherent damped oscillations about the global minimum of the potential. The rate of energy density loss, due to the expansion of the universe, is given by

$$
\dot{\rho}=-3 H \dot{\phi}^{2}=-3 H(\rho+p)=-3 H \gamma \rho,
$$

where $\rho=\dot{\phi}^{2} / 2+V(\phi), p=\dot{\phi}^{2} / 2-V(\phi)$, and we averaged $p$ over one oscillation of $\phi$. From this equation and Eq. (2.5), we obtain

$$
\rho \propto a^{-3 \gamma}, \quad a(t) \propto t^{\frac{2}{3 \gamma}} .
$$

The parameter $\gamma$ depends on the shape of the potential $V(\phi)$. For a quadratic (quartic) potential, $\gamma=1(\gamma=4 / 3)$ and the expansion is similar to that of a matter (radiation) dominated universe.

In order to discuss the subsequent decay of the inflaton, we must use its full evolution equation in Eq. (6.2), which also includes the decay term $\Gamma_{\phi} \dot{\phi}$. This is approximately solved $[12,50]$ by

$$
\rho(t)=\rho_{\mathrm{f}}\left(\frac{t}{t_{\mathrm{f}}}\right)^{-2} e^{-\Gamma_{\phi} t}
$$

where $\rho_{\mathrm{f}}$ is the energy density at $t_{\mathrm{f}}$ and, for definiteness, we considered that the potential is quadratic. The second and third factors in the RHS of this equation represent the dilution of the field energy due to the expansion of the universe and the decay of $\phi$ to light particles respectively.

All pre-existing radiation (known as old radiation) was diluted by inflation, so the only radiation present is the one produced by the decay of $\phi$ and is known as new radiation. Its energy density $\rho_{\mathrm{r}}$ satisfies $[12,50]$ the equation

$$
\dot{\rho}_{\mathrm{r}}=-4 H \rho_{\mathrm{r}}+\gamma \Gamma_{\phi} \rho,
$$

where the first term in the RHS represents the dilution of radiation due to the cosmological expansion while the second one is the energy density transfer from $\phi$ to radiation. This yields

$$
\rho_{\mathrm{r}}=\frac{3}{5} \rho \Gamma_{\phi} t\left[1+\frac{3}{8} \Gamma_{\phi} t+\frac{9}{88}\left(\Gamma_{\phi} t\right)^{2}+\cdots\right]
$$


with $\rho=\rho_{\mathrm{f}}\left(t / t_{\mathrm{f}}\right)^{-2} \exp \left(-\Gamma_{\phi} t\right)$ being the energy density of the field $\phi$. Initially, $\rho_{\mathrm{r}} \ll \rho$, but, at $t_{\mathrm{d}}=\Gamma_{\phi}^{-1}, \rho_{\mathrm{r}}$ dominates and the universe becomes radiation dominated. The temperature at $t_{\mathrm{d}}$ is

$$
T_{\mathrm{r}}=\left(\frac{45}{2 \pi^{2} g_{*}}\right)^{\frac{1}{4}}\left(\Gamma_{\phi} m_{\mathrm{P}}\right)^{\frac{1}{2}}
$$

and is historically called reheat temperature although there is neither supercooling nor reheating.

As already mentioned, inflation not only homogenizes the universe but also generates the density perturbations needed for structure formation. An important notion for understanding the underlying mechanism is event horizon. It includes all points with which we will eventually communicate sending signals at $t$. In contrast to the case of matter or radiation dominance, the instantaneous radius of the event horizon during inflation is finite:

$$
d_{e}(t)=a(t) \int_{t}^{\infty} \frac{d t^{\prime}}{a\left(t^{\prime}\right)}=H^{-1}<\infty
$$

Points in our event horizon at $t$ are eventually pulled out by the exponential expansion. We say that these points (and the corresponding scales) crossed outside the event horizon. Actually, the situation is like in a black hole turned inside out. Then, exactly as in a black hole, there are quantum fluctuations of the thermal type governed by the Hawking temperature [51] $T_{\mathrm{H}}=H / 2 \pi$. It turns out [52] that the quantum fluctuations of the inflaton are $\delta \phi=T_{\mathrm{H}}$ leading to density perturbations $\delta \rho=$ $V^{\prime}(\phi) \delta \phi$. As the scale $\ell$ of these perturbations crosses outside the event horizon, they become [53] classical. When $\ell$ ( or $k=2 \pi / \ell$ ) re-enters the post-inflationary horizon, we obtain the perturbation [54] (for a review, see e.g. Ref. [55])

$$
\left(\frac{\delta \rho}{\rho}\right)_{\ell}=\frac{1}{5 \sqrt{3} \pi} \frac{V^{\frac{3}{2}}\left(\phi_{\ell}\right)}{m_{\mathrm{P}}^{3} V^{\prime}\left(\phi_{\ell}\right)},
$$

where $\phi_{\ell}$ is the value of $\phi$ when $\ell$ crosses outside the horizon. From the results of COBE [4], $(\delta \rho / \rho) \simeq 2 \times 10^{-5}$ at the pivot scale $k=0.002 \mathrm{Mpc}^{-1}$. The spectrum of the perturbations $\delta \rho / \rho \propto$ $\ell^{\alpha_{\mathrm{s}}}$ is characterized by the scalar spectral index $n_{\mathrm{s}}=1-2 \alpha_{\mathrm{s}}=1+2 \eta-6 \varepsilon$. The Harrison-Zeldovich flat spectrum [45] corresponds to $n_{\mathrm{s}}=1$, while the experimental value is $n_{\mathrm{s}}=0.968 \pm 0.006$ [6].

The density fluctuations on the last scattering surface produce temperature fluctuations in the CMBR. The dominant mechanism is the scalar Sachs-Wolfe [56] effect: regions with a deep gravitational potential will cause the photons to lose energy as they climb up the potential well and, thus, appear cooler. The scalar quadrupole anisotropy is

$$
\left(\frac{\delta T}{T}\right)_{\mathrm{S}}=\frac{1}{12 \sqrt{5} \pi} \frac{V^{\frac{3}{2}}\left(\phi_{\ell}\right)}{m_{\mathrm{P}}^{3} V^{\prime}\left(\phi_{\ell}\right)} .
$$

There are also tensor fluctuations [57] in the temperature of the CMBR with the tensor quadrupole anisotropy given by

$$
\left(\frac{\delta T}{T}\right)_{\mathrm{T}} \simeq 0.15 \frac{V^{\frac{1}{2}}\left(\phi_{\ell}\right)}{m_{\mathrm{P}}^{2}}
$$

The tensor-to-scalar ratio

$$
r=\frac{(\delta T / T)_{T}^{2}}{(\delta T / T)_{S}^{2}}
$$

is experimentally bound to be $\lesssim 0.06[6]$. 


\section{Baryogenesis}

A successful inflationary scenario has to be followed by a successful reheating process [12]. Note that, in the case of supersymmetric theories, the reheat temperature $T_{\mathrm{r}} \lesssim 10^{9} \mathrm{GeV}$ since otherwise the gravitino relic density is unacceptably large [14]. Also the observed [6] baryon asymmetry $n_{\mathrm{B}} / s \simeq 8.66 \times 10^{-11}[6]$ should be generated after inflation $\left(n_{\mathrm{B}}\right.$ and $s$ are the baryon number and entropy densities). The most promising way to generate the baryon asymmetry after inflation is via non-thermal [15, 58] leptogenesis [16] (for a review, see Ref. [59]). In accordance with this scenario, the inflaton decays into a pair of right handed neutrinos $\left(v^{c}\right)$. The subsequent out-ofequilibrium decay of these $v^{c}$ 's into (anti)leptons and electroweak Higgs fields can generate a primordial lepton asymmetry provided there is CP-violation. This asymmetry, at the electroweak transition, is partly turned [17] into baryon asymmetry by sphaleron [18] effects.

In order to understand the sphaleron mechanism, let us recall that the vacua of the $S U(2)_{\mathrm{L}}$ gauge theory are characterized [60] by a winding number $n \in Z$. In the presence of the VEV of the electroweak doublet which breaks $S U(2)_{\mathrm{L}}$, the minimal height of the potential barrier between the vacua with winding numbers $n$ and $n+1$ corresponds to a static solution [61] called sphaleron, which is actually a saddle point of the potential. The mass of the sphaleron is about $10-15 \mathrm{TeV}$. At $T=0$, the tunneling between the vacua $|n\rangle$ and $|n+1\rangle$ is utterly suppressed. However, between $T \sim 200 \mathrm{GeV}$ and the critical temperature $T_{\mathrm{c}}$ of the electroweak phase transition, this tunneling is very frequent and in equilibrium with the universe $[17,18]$. Baryon $(B)$ and Lepton $(L)$ number anomalies imply [62] that the tunneling from $|n\rangle$ to $|n+1\rangle$ is accompanied by a change $\Delta B=$ $\Delta L=-3$. Note that $B-L$ is not violated and the primordial asymmetry in $-L$ can be considered as primordial $B-L$ asymmetry, which remains constant at subsequent times. At the electroweak phase transition this asymmetry is partly converted into $B$ asymmetry. The resulting $n_{\mathrm{B}} / s$ can be calculated [63] by setting equal to zero the algebraic sum of the chemical potentials $(\mu)$ of all the particles involved in each of the reactions which are in thermal equilibrium. Solving the system of the resulting equations, we find the fraction of the primordial $B-L$ asymmetry which turns into $B$ asymmetry.

\section{Dark Energy}

Inflation implies that the present universe is exactly flat $\left(\Omega=\rho / \rho_{\mathrm{c}}=1\right)$ and thus will keep expanding for ever. This is also confirmed by the recent measurements [6], which also reveal that matter is only $27 \%$ of the universe. The question then arises what is the rest $73 \%$ of the universe made of. The answer to this question was first given in 1997-8 by the observations on Supernovae Ia [64], which are seen today as they were in a previous cosmic time. The unexpected result of these observations was that the expansion of the universe in previous times was slower than it is today, i.e. the expansion of the universe is accelerating. This can be explained if more than $2 / 3$ of the energy in the universe is in the form of cosmological constant, i.e. it is practically not diluted by the expansion exactly as the energy driving inflation (because of the existence of negative pressure close in magnitude to the energy density). One can then wonder whether we are about to enter a new inflationary phase. The idea of quintessence [65] (for a review, see Ref. [66]) though tells us that this may not be the case. The WMAP and Planck satellites $[5,6]$ confirm that the $73 \%$ of the 
energy density in the universe is in the form of dark energy, i.e. a form close to a cosmological constant.

\section{Dark Matter}

Studies of nucleosynthesis [1] combined with the results of the Planck satellite [6] imply that the baryonic (visible) matter constitutes only $4.85 \%$ of the energy density in the universe. Therefore, if one subtracts baryonic matter from the total matter content of the universe, which is about $27 \%$, concludes that about $22 \%$ of the universe consists of dark matter. The question then arises what is the nature of dark matter. Dark matter is usually considered to be cold, i.e. consisting of non-relativistic massive particles which interact very weakly (mainly gravitationally) with all other particles. However, very light weakly interacting particles such as axions can also contribute to dark matter since they are non-relativistic for different reasons. Also warm or hot dark matter has been considered.

What could the dark matter particle be? There are several proposal, but I will mention only the most important ones.

1. Lightest supersymmetric particle (LSP): This particle is the most promising candidate for dark matter [19] since it is protected by a discrete $Z_{2} R$-parity symmetry from decaying into lighter particles. The LSPs can annihilate in pairs or coannihilate with the next-to-LSPs. There are many detailed calculations of the relic density of the LSPs (see e.g. Refs. [67, 68]) which show that, under certain circumstances, they can account for the dark matter.

2. Axion: This light boson is connected with the Peccei-Quinn solution [22] of the strong CP problem and can contribute [69] to dark matter. However, one has to be careful since, if combined with inflation, it may lead to unacceptably large isocurvature perturbations [70] in the CMBR. This problem is avoided if the Peccei-Quinn symmetry breaking occurs after the end of inflation [24] or if the value of the Peccei-Quinn field during inflation is kept large [71].

3. Superheavy fermions: Intermediate scale dark matter particles coexisting with axions appear in Refs. [24, 25], while superheavy dark matter particles (wimpzillas) have been studied in Ref. [23].

The LSPs could be detected by their scattering off nuclei as in the XENON 1T experiment [72], which has no positive results so far. The detection of axions is even harder. In supersymmetric theories the gravitino [73], the superpartner of graviton, or the axino [74] can also contribute to the composition of dark matter.

The most promising candidate for dark matter is the LSP. In supersymmetric theories, there exist a discrete $Z_{2}$ symmetry known as R-parity, which is necessary for preventing unacceptably fast proton decay. Under R-parity, all the standard model particles are even, while all the supersymmetric particles are odd. By virtue of R-parity, the LSP, which is normally the lightest neutralino, is stable as it cannot decay to lighter particles and thus is a good candidate for dark matter. The supersymmetric particles can annihilate into pairs to standard model particles. To find the LSP 
relic abundance, we need to consider also coannihilations of the LSP with the next-to-LSP, which is usually the lightest stau. This is a very complicated calculation since very many processes are involved but, fortunately, there are already publicly available codes for this calculation such as microMEGAs [68]. The relic density of neutralinos usually comes out too large. However, the following main mechanisms can reduce the LSP relic abundance to the observed value of dark matter abundance $\Omega_{\mathrm{DM}} h^{2} \simeq 0.12$ [6].

1. A-pole exchange: The LSP pair annihilation can be resonantly enhanced by an A-pole exchange in the $s$-channel [20] ( $A$ is the CP-odd Higgs boson in the minimal supersymmetric standard model).

2. Coannihilation: Coannihilation [21] of the LSP with the next-to-LSP, which is usually the lightest stau eigenstate, can reduce drastically the relic LSP density. This, however, requires a small mass splitting between the LSP and the next-to-LSP.

\section{Quantum Gravity}

So far, we considered gravity at the classical (non-quantum) level and separately from the other three interactions, which are described by relativistic quantum field theory and possibly unified in a GUT. The questions then arise:

(a) Can gravity be quantized as the other three interactions?

(b) Can it be unified with the other three interactions to yield the theory of everything?

Quantum gravity phenomena are expected to appear at very high energies of the order of the Planck mass $m_{\mathrm{P}} \simeq 2.44 \times 10^{18} \mathrm{GeV}$, or at small distances of the order of the Planck length $\ell_{\mathrm{P}} \equiv m_{\mathrm{P}}^{-1} \sim$ $10^{-33} \mathrm{~cm}$, or at small times of the order of $t_{\mathrm{P}} \sim 10^{-43} \mathrm{sec}$. Therefore, quantum fluctuations of gravity are expected to be important

(i) very near the big bang, i.e. for cosmic times $t \lesssim t_{\mathrm{P}}$, and

(ii) for the possible generation of primordial gravity waves, which could originate from the quantum fluctuations of gravity during cosmological inflation. These waves may be detected in the future by the space based observatory LISA.

In trying to construct a quantum field theory for gravity, we encounter problems with renormalizability. In order to understand the meaning of renormalizability, let us consider classical electrodynamics, as an example. Relativity implies that every elementary particle must be pointlike. Consequently, the energy of the electric field $\overline{\mathbf{E}}$ around such a particle with charge $e$ (e.g. the electron) is proportional to

$$
\int_{0}^{\infty} \overline{\mathbf{E}}^{2} 4 \pi r^{2} d r=4 \pi e^{2} \int_{0}^{\infty} \frac{d r}{r^{2}}
$$

This integral diverges at $r=0$ and thus the energy is infinite. We must then assume that the bare mass of the particle is $-\infty$, so that the final mass is $\infty-\infty$ and turns out to be finite. 
This situation appears also in quantum electrodynamics, where we encounter several meaningless infinities while performing various calculations. However, all these infinities can be systematically gathered and put inside two parameters: the mass and charge of the electron. We then take the bare values of these parameters to be infinite, so that their final renormalized values are finite and equal to their experimental values. This complicated mathematical procedure is called renormalization and the theories where it applies are called renormalizable. The three interactions (except gravity) are renormalizable as shown by 't Hooft and Veltman (for a review, see Ref. [75]). Therefore, with a finite number of experiments, we can determine the values of a finite number of parameters and then everything else can be predicted. So, the theory has a high predictability. The problem with quantum gravity is that, as it turns out, it is not renormalizable.

\section{String Theory}

The theory of (super)strings [26] was proposed in order to cure the non-renormalizabity of gravity and also unify it with the other three interactions, so as to achieve the construction of the theory of everything. The idea is that the fundamental objects are not point-like particles, but one dimensional strings. Adding supersymmetry to the scheme one obtains superstrings. We have strings of size $\ell_{\mathrm{P}} \sim 10^{-33} \mathrm{~cm}$ which vibrate, but for the present energies look like point particles. The various vibrational modes of these strings appear as particles with different quantum numbers and we obtain a unified description of all particles and all their interactions. The abandonment of point particles removes the notorious infinities. It is actually believed that string theory gives renormalizable or even completely finite unification of all four interactions (see e.g. Ref. [76]). One of the vibrational modes is the graviton, which is the carrier of gravitational interactions. Therefore, we can, in principle, describe quantum gravity and the other three interactions in a unified way, which would be the theory of everything.

However, there are some important shortcomings of string theory.

(i) Initially, people thought that there is a unique solution of string theory, which would make it of very high predictability. However, we now know that there exists a huge number of solutions $\left(\sim 10^{500}\right)$. The string vacua comprise a collective landscape [77] with a huge number of hills, valleys, etc and, therefore, a huge number of minima (solutions). One can then employ the so called anthropic principle, which states that the minimum (solution) corresponding to our universe had the right conditions so as to produce us eventually. In my opinion, this is not an acceptable scientific way of thinking.

(ii) Although there exist very many solutions with a variety of discrete symmetries (see e.g. Ref. [78]), none of them reproduces exactly our universe.

An important prediction of string theory is that there exist ten spacetime dimensions [26] (or eleven in the case of $M$ theory [79]). Six of them are compactified, i.e. they are strongly curved to form a 6-dimensional compact manifold of size $\ell_{\mathrm{P}} \sim 10^{-33} \mathrm{~cm}$. The other four dimensions remain open and correspond to the usual spacetime dimensions. The geometric structure of the compactified dimensions determines many of the phenomena encountered in the 4-dimensional spacetime. 
As already mentioned strings allow us to discuss quantum gravity and thus give us the possibility to approach the big bang, i.e. the initial singularity at $t=0$. Therefore, we can consider cosmic times $t \lesssim t_{\mathrm{P}} \sim 10^{-43} \mathrm{sec}$ or even negative times before the big bang. One problem we can address, in the light of string theory, is the problem of initial conditions [27] for inflation.

Conventional inflation takes place at the GUT phase transition at cosmic time $t \sim 10^{-37} \mathrm{sec} \gg$ $t_{\mathrm{P}}$, where we need, as initial condition for inflation to start, a large region of size few times $H^{-1}$ within which the fields are homogenized taking appropriate values [80]. However, this region consists of many regions of smaller size $\ell$, which resulted from the expansion of regions of size $\ell_{\mathrm{P}}$ at the Planck time, where causal communication could not exceed the distance $\ell_{\mathrm{P}}$. Therefore, a region of size $H^{-1}$ at the onset of inflation cannot be homogenized. This is again a problem of initial conditions. Its resolution requires a first stage of inflation near or before the big bang to provide the necessary homogenization for the onset of conventional inflation. One possible solution could be the pre-big-bang scenario [81]. Imagine that we travel backwards in time and pass through the initial singularity at $t=0$, which is though smoothed by strings, and enter the realm of negative times (before the big bang). There, during the motion of the universe towards the initial singularity, we have conditions of very high curvature and the extra dimensions contract and compactify. This causes inflation (accelerated expansion) in the four open dimensions. This can be a first stage of inflation providing the initial conditions for the conventional inflation or can be the main inflation producing the density perturbations too. We should be though very careful with these stringy considerations since the physics of strings is not fully understood or solved yet. Note that the problem of initial conditions for inflation can be solved by a conventional two stage inflation [82] too.

Another application of quantum gravity could be the explanation of the origin of primordial gravity waves if such waves are detected. The BICEP2 experiment [83] observed the polarization vector pattern of the CMBR, which can be split into an $\overline{\mathbf{E}}$-mode resembling an electrostatic field with sources and sinks and a $\overline{\mathbf{B}}$-mode resembling a magnetostatic field with vortices. Subtracting the $\overline{\mathbf{B}}$-mode from polarized dust from the observed $\overline{\mathbf{B}}$-mode in CMBR, they found that there is a remaining $\overline{\mathbf{B}}$-mode, which could be due to primordial gravity waves from inflation. The subsequent Planck satellite measurements [6], however, did not confirm this conclusion, which as it turned out underestimated the foreground from polarized dust. However, possible existence of primordial gravity waves, which may be measurable in the foreseeable future, cannot be excluded since the Planck measurements [6] put only a moderate upper bound on the tensor-to-scalar ratio $r \lesssim 0.06$. These waves could originate from tensor (gravitational) quantum fluctuations during inflation which become classical fluctuation (i.e. gravity waves) as they exit the inflationary horizon. Therefore, quantum gravity may be required to understand the origin of these waves.

\section{Conclusions}

We presented the SBB cosmological model together with its successes and shortcomings, which are resolved by inflation, i.e. a period of exponential expansion in the early stages of the universe evolution. This may have happened during the GUT phase transition at which the relevant Higgs field was displaced from its vacuum value. This field (inflaton) could then, for some time, roll slowly towards the vacuum providing an almost constant false vacuum energy density driving 
the exponential expansion. Inflation provides the density perturbations required for the generation of the large scale structure in the universe and the temperature fluctuations in the CMBR. After the end of inflation, the inflaton performs damped oscillations about the vacuum, decays, and reheats the universe.

We described how the observed baryon asymmetry of the universe is generated, in inflationary models, via non-thermal leptogenesis. The inflaton normally decays into right handed neutrinos, whose subsequent out-of-equilibrium decay into leptons (antileptons) and electroweak Higgs fields produces a primordial lepton asymmetry. This asymmetry is then partly converted into baryon asymmetry by the electroweak sphaleron effects. Recent measurements revealed that the present universe is flat with matter constituting only $27 \%$ of its energy density. The rest $73 \%$ of the universe is in the form of dark energy. On the other hand, the baryonic (visible) matter constitutes only $4.85 \%$ of the universe. The rest $22 \%$ is made of dark matter. The most promising candidate for dark matter is the LSP since it is protected by a discrete $Z_{2}$ R-parity symmetry from decaying. These particles can annihilate in pairs or coannihilate with the next-to-LSPs and their relic density can be reduced to the observed value of dark matter abundance mainly by resonant pair annihilation or coannihilation if they are almost degenerate with the next-to-LSPs. Other possible dark matter candidates include the axion and superheavy or intermediate scale fermions.

We sketched briefly the renormalization problem encountered in trying to quantize gravity and its possible resolution by string theory, whose aim is not only to construct a viable theory for quantum gravity but also to unify it with the other three interactions. The main disadvantage of string theory is that it admits a huge number of solutions, but none of them seems to reproduce exactly our universe. It predicts that there exist ten spacetime dimensions (or eleven in the case of M theory). Six of them are compactified, while the other four remain open and are the usual spacetime dimensions. The geometric structure of the compactified dimensions determines many of the phenomena in the 4-dimensional spacetime.

String theory allows us to discuss physics very near or even before the big bang, where the quantum fluctuations of gravity are present. Therefore, it can help in resolving the problem of initial conditions for inflation. In order to have inflation started, one needs a large homogeneous region around the time of the GUT phase transition. However, this region consists of many smaller regions which originate from homogenized regions around the Planck era, where the causal communication could not extend to distances larger than the Planck length. Therefore, a first stage of inflation near or before the big bang which provides the required homogenization at the onset of inflation is needed. Such a primordial inflation can take place in the pre-big-bang period. During the motion of the universe towards the initial singularity, we have conditions of very high curvature and the extra dimensions contract and compactify. This causes inflation in the four open dimensions. Another application of quantum gravity could be the explanation of the origin of primordial gravity waves from inflation if such waves are detected in the future, which is not excluded by the recent data.

\section{References}

[1] G. Steigman, Primordial nucleosynthesis: successes and challenges, Int. J. Mod. Phys. E 15 (2006) 1.

[2] S. Weinberg, Gravitation and Cosmology, J. Wiley and Sons, New York 1972; E.W. Kolb and M.S. Turner, The Early Universe, Front. Phys. 69 (1990) 1. 
[3] J.C. Pati and A. Salam, Is Baryon Number Conserved?, Phys. Rev. Lett. 31 (1973) 661; H. Georgi and S. Glashow, Unity of All Elementary Particle Forces, Phys. Rev. Lett. 32 (1974) 438.

[4] C.L. Bennett et al., 4-Year COBE DMR Cosmic Microwave Background Observations: Maps and Basic Results, Astrophys. J. 464 (1996) L1.

[5] G. Hinshaw et al., Nine-Year Wilkinson Microwave Anisotropy Probe (WMAP) Observations: Cosmological Parameter Results, Astrophys. J. Suppl. 208 (2013) 19.

[6] N. Aghanim et al., Planck 2018 results. VI. Cosmological parameters, arXiv:1807.06209 [astro-ph.CO].

[7] G.'t Hooft, Magnetic Monopoles in Unified Gauge Theories, Nucl. Phys. B 79 (1974) 276; A. Polyakov, Particle Spectrum in the Quantum Field Theory, JETP Lett. 20 (1974) 194.

[8] J.P. Preskill, Cosmological Production of Superheavy Magnetic Monopoles, Phys. Rev. Lett. 43 (1979) 1365.

[9] P.J.E. Peebles, The Large-Scale Structure of the Universe, Princeton University Press, Princeton 1980; G. Efstathiou, The Physics of the Early Universe, in proceedings of the 36th Scottish Universities Summer School in Physics, Editors J.A. Peacock et al., Adam-Higler, Bristol 1990.

[10] A.H. Guth, The Inflationary Universe: A Possible Solution to the Horizon and Flatness Problems, Phys. Rev. D 23 (1981) 347.

[11] G. Lazarides, Introduction to cosmology, in proceedings of Corfu Summer Institute on Elementary Particle Physics, 1998 PoS(corfu98)014 [hep-ph/9904502]; A.R. Liddle and D.H. Lyth, Cosmological Inflation and Large-Scale Structure, Cambridge University Press, Cambridge 2000; G.S. Watson, An exposition on inflationary cosmology, astro-ph/0005003; G. Lazarides, Inflationary cosmology, Lect. Notes Phys. 592 (2002) 351 [hep-ph/0111328]; G. Lazarides, Introduction to inflationary cosmology, in proceedings of Corfu Summer Institute on Elementary Particle Physics, 2001 [hep-ph/0204294]; V.A. Rubakov, Introduction to Cosmology, in proceedings of RTN Winter School on Strings, Supergravity and Gauge Theories, SISSA 2005 PoS(RTN2005)003; A.R. Liddle and D.H. Lyth, The primordial density perturbation: Cosmology, inflation and the origin of structure, Cambridge University Press, Cambridge 2009.

[12] R.J. Scherrer and M.S. Turner, Decaying Particles Do Not Heat Up the Universe, Phys. Rev. D 31 (1985) 681.

[13] G. Lazarides, C. Panagiotakopoulos, and Q. Shafi, Relaxing the Cosmological Bound on Axions, Phys. Lett. B 192 (1987) 323; G. Lazarides, R.K. Schaefer, D. Seckel, and Q. Shafi, Dilution of Cosmological Axions by Entropy Production, Nucl. Phys. B 346 (1990) 193.

[14] M.Yu. Khlopov and A.D. Linde, Is it Easy to Save the Gravitino?, Phys. Lett. B 138 (1984) 265; J.R. Ellis, J.E Kim, and D.V. Nanopoulos, Cosmological Gravitino Regeneration and Decay, Phys. Lett. B 145 (1984) 181; J.R. Ellis, D.V. Nanopoulos, and S. Sarkar, The Cosmology of Decaying Gravitinos, Nucl. Phys. B 259 (1985) 175; J.R. Ellis, G.B. Gelmini, J.L. López, D.V. Nanopoulos, and S. Sarkar, Astrophysical constraints on massive unstable neutral relic particles, Nucl. Phys. B 373 (1992) 399; M. Kawasaki and T. Moroi, Gravitino production in the inflationary universe and the effects on big bang nucleosynthesis, Prog. Theor. Phys. 93 (1995) 879.

[15] G. Lazarides and Q. Shafi, Origin of matter in the inflationary cosmology, Phys. Lett. B 258 (1991) 305; G. Lazarides, R.K. Schaefer, and Q. Shafi, Supersymmetric inflation with constraints on superheavy neutrino masses, Phys. Rev. D 56 (1997) 1324. 
[16] M. Fukugita and T.T. Yanagida, Baryogenesis Without Grand Unification, Phys. Lett. B 174 (1986) 45.

[17] S. Dimopoulos and L. Susskind, On the Baryon Number of the Universe, Phys. Rev. D 18 (1978) 4500.

[18] V.A. Kuzmin, V.A. Rubakov, and M. Shaposhnikov, On the Anomalous Electroweak Baryon Number Nonconservation in the Early Universe, Phys. Lett. B 155 (1985) 36; P. Arnold and L. McLerran, Sphalerons, Small Fluctuations and Baryon Number Violation in Electroweak Theory, Phys. Rev. D 36 (1987) 581.

[19] H. Goldberg, Constraint on the Photino Mass from Cosmology, Phys. Rev. Lett. 50 (1983) 1419; J.R. Ellis, J.S. Hagelin, D.V. Nanopoulos, K.A. Olive, and M. Srednicki, Supersymmetric Relics from the Big Bang, Nucl. Phys. B 238 (1984) 453.

[20] V. Barger and C. Kao, Relic density of neutralino dark matter in supergravity models, Phys. Rev. D 57 (1998) 3131; J.R. Ellis and K.A. Olive, Revisiting the Higgs Mass and Dark Matter in the CMSSM, Eur. Phys. J. C 72 (2012) 2005.

[21] K. Griest and D. Seckel, Three exceptions in the calculation of relic abundances, Phys. Rev. D 43 (1991) 3191.

[22] R.D. Peccei and H.R. Quinn, CP Conservation in the Presence of Instantons, Phys. Rev. Lett. 38 (1977) 1440; S. Weinberg, A New Light Boson?, Phys. Rev. Lett. 40 (1978) 223; F. Wilczek, Problem of Strong P and T Invariance in the Presence of Instantons, Phys. Rev. Lett. 40 (1978) 279.

[23] E.W. Kolb, D.J.H. Chung, and A. Riotto, WIMPzillas!, AIP Conf. Proc. 484 (1999) 91 [hep-ph/9810361].

[24] G. Lazarides and Q. Shafi, Axion Model with Intermediate Scale Fermionic Dark Matter, arXiv: 2004.11560 [hep-ph].

[25] G. Lazarides and Q. Shafi, Monopoles, axions and intermediate mass dark matter, Phys. Lett. B 489 (2000) 194.

[26] M.B. Green and J.H. Schwarz, Anomaly Cancellation in Supersymmetric D $=10$ Gauge Theory and Superstring Theory, Phys. Lett. B 149 (1984) 117; D.J. Gross, J.A. Harvey, E.J. Martinec, and R. Rohm, The Heterotic String, Phys. Rev. Lett. 54 (1985) 502; M. Dine, V. Kaplunovsky, M.L. Mangano, C. Nappi, and N. Seiberg, Superstring Model Building, Nucl. Phys. B 259 (1985) 549; E. Witten, Symmetry Breaking Patterns in Superstring Models, Nucl. Phys. B 258 (1985) 75; P. Candelas, G.T. Horowitz, A. Strominger, and E. Witten, Vacuum Configurations for Superstrings, Nucl. Phys. B 258 (1985) 46.

[27] D.S. Goldwirth and T. Piran, Initial conditions for inflation, Phys. Reports 214 (1992) 223.

[28] D.A. Kirzhnits and A.D. Linde, Macroscopic Consequences of the Weinberg Model, Phys. Lett. B 42 (1972) 471.

[29] S.L. Glashow, Trinification of All Elementary Particle Forces, in Fifth Workshop on Grand Unification, edited by K. Kang, H.M. Fried, and P.H. Frampton, World Scienific, Singapore 1984; K.S. Babu, X.-G. He, and S. Pakvasa, Neutrino Masses and Proton Decay Modes in $S U(3) \times S U(3) \times S U(3)$ Trinification, Phys. Rev. D 33 (1986) 763; G. Lazarides, C. Panagiotakopoulos, MSSM from SUSY trinification, Phys. Lett. B 336 (1994) 190; S. Willenbrock, Triplicated trinification, Phys. Lett. B 561 (2003) 130; J. Sayre, S. Wiesenfeldt, and S. Willenbrock, Minimal trinification, Phys. Rev. D 73 (2006) 035013. 
[30] M. Yoshimura, Unified Gauge Theories and the Baryon Number of the Universe, Phys. Rev. Lett. 41 (1978) 281, (E) 42 (1979) 746; A.Yu Ignatiev, N.V. Krasnikov, V.A. Kuzmin, and A.N. Tavkhelidze, Universal CP Noninvariant Superweak Interaction and Baryon Asymmetry of the Universe, Phys. Lett. B 76 (1978) 436; J.R. Ellis, M.K. Gaillard, and D.V. Nanopoulos, Baryon Number Generation in Grand Unified Theories, Phys. Lett. B 80 (1978) 360, (E) 82 (1979) 464; D. Toussaint, S.B. Treiman, F. Wilczek, and A. Zee, Matter-Antimatter Accounting, Thermodynamics, and Black Hole Radiation, Phys. Rev. D 19 (1979) 1036; S. Weinberg, Cosmological Production of Baryons, Phys. Rev. Lett. 42 (1979) 850.

[31] T.W.B. Kibble, Topology of Cosmic Domains and Strings, J. Phys. A 9 (1976) 1387; T.W.B. Kibble, Some implications of a cosmological phase transition, Phys. Reports 67 (1980) 183.

[32] H.B. Nielsen and P. Olesen, Vortex Line Models for Dual Strings, Nucl. Phys. B 61 (1973) 45.

[33] Ya.B. Zeldovich, I.Yu. Kobzarev, and L.B. Okun, Cosmological Consequences of the Spontaneous Breakdown of Discrete Symmetry, JETP (Sov. Phys.) 40 (1975) 1.

[34] G. Lazarides, C. Panagiotakopoulos, and Q. Shafi, Baryogenesis and the Gravitino Problem in Superstring Models, Phys. Rev. Lett. 56 (1986) 557; G. Lazarides and Q. Shafi, Anomalous discrete symmetries and the domain wall problem, Nucl. Phys. B 392 (1993) 61; D.H. Lyth and E.D. Stewart, Cosmology with a TeV mass GUT Higgs, Phys. Rev. Lett. 75 (1995) 201; D.H. Lyth and E.D. Stewart, Thermal inflation and the moduli problem, Phys. Rev. D 53 (1996) 1784.

[35] T.W.B. Kibble, G. Lazarides, and Q. Shafi, Strings in SO(10), Phys. Lett. B 113 (1982) 237.

[36] X. Siemens, V. Mandic, and J. Creighton, Gravitational wave stochastic background from cosmic (super)strings, Phys. Rev. Lett. 98 (2007) 111101; M.R. DePies and C.J. Hogan, Stochastic Gravitational Wave Background from Light Cosmic Strings, Phys. Rev. D 75 (2007) 125006; S. Kuroyanagi, K. Miyamoto, T. Sekiguchi, K. Takahashi, and J. Silk, Forecast constraints on cosmic string parameters from gravitational wave direct detection experiments, Phys. Rev. D 86 (2012) 023503; J.J. Blanco-Pillado and K.D. Olum, Stochastic gravitational wave background from smoothed cosmic string loops, Phys. Rev. D 96 (2017) 104046; J.J. Blanco-Pillado, K.D. Olum, and X. Siemens, New limits on cosmic strings from gravitational wave observation, Phys. Lett. B 778 (2018) 392.

[37] G. Lazarides and C. Panagiotakopoulos, Gravitational Waves from Double Hybrid Inflation, Phys. Rev. D 92 (2015) 123502.

[38] G. Lazarides and Q. Shafi, Monopoles, Strings, and Necklaces in SO(10) and E 6 , J. High Energy Phys. 10 (2019) 193.

[39] G. Lazarides and Q. Shafi, Axion Models with No Domain Wall Problem, Phys. Lett. B 115 (1982) 21.

[40] T.W.B. Kibble, G. Lazarides, and Q. Shafi, Walls Bounded by Strings, Phys. Rev. D 26 (1982) 435.

[41] G. Lazarides, Q. Shafi, and T.F. Walsh, Cosmic Strings and Domains in Unified Theories, Nucl. Phys. B 195 (1982) 157.

[42] P.J.E. Peebles, D.N. Schramm, E.L. Turner, and R.N. Kron, The Case for the hot big bang cosmology, Nature 352 (1991) 769.

[43] S. Burles, K.M. Nollett, and M.S. Turner, Big bang nucleosynthesis predictions for precision cosmology, Astrophys. J. 552 (2001) 1.

[44] M.B. Einhorn, The Production of Magnetic Monopoles in the Very Early Universe, in proceedings of Unification of the Fundamental Particle Interactions, Erice 1980, Editors S. Ferrara et al, Plenum Press, New York 1980. 
[45] E.R. Harrison, Fluctuations at the threshold of classical cosmology, Phys. Rev. D 1 (1970) 2726; Ya.B. Zeldovich, A Hypothesis unifying the structure and the entropy of the universe, Mon. Not. Roy. Astr. Soc. 160 (1972) 1.

[46] G. Lazarides and Q. Shafi, Extended Structures at Intermediate Scales in an Inflationary Cosmology, Phys. Lett. B 148 (1984) 35; G. Lazarides, C. Panagiotakopoulos and Q. Shafi, Magnetic monopoles from superstring models, Phys. Rev. Lett. 58 (1987) 1707.

[47] A.D. Dolgov and A.D. Linde, Baryon Asymmetry in Inflationary Universe, Phys. Lett. B 116 (1982) 329; L.F. Abbott, E. Farhi, and M.B. Wise, Particle Production in the New Inflationary Cosmology, Phys. Lett. B 117 (1982) 29.

[48] A.D. Dolgov and S.H. Hansen, Equation of motion of a classical scalar field with back reaction of produced particles, Nucl. Phys. B 548 (1999) 408.

[49] P.J. Steinhardt and M.S. Turner, A Prescription for Successful New Inflation, Phys. Rev. D 29 (1984) 2162.

[50] M.S. Turner, Coherent Scalar Field Oscillations in an Expanding Universe, Phys. Rev. D 28 (1983) 1243.

[51] S.W. Hawking, Black hole explosions?, Nature 248 (1974) 30; S.W. Hawking, Particle Creation by Black Holes, Commun. Math. Phys. 43 (1975) 199, (E) 46 (1976) 206; G.W. Gibbons and S.W. Hawking, Action Integrals and Partition Functions in Quantum Gravity, Phys. Rev. D 15 (1977) 2752.

[52] T. Bunch and P.C.W. Davies, Quantum field theory in de Sitter space: Renormalization by point-splitting, Proc. Roy. Soc. London A 360 (1978) 117; A. Vilenkin and L.H. Ford, Gravitational Effects upon Cosmological Phase Transitions, Phys. Rev. D 26 (1982) 1231; A.D. Linde, Scalar Field Fluctuations in Expanding Universe and the New Inflationary Universe Scenario, Phys. Lett. B 116 (1982) 335; A.A. Starobinsky, Dynamics of Phase Transition in the New Inflationary Universe Scenario and Generation of Perturbations, Phys. Lett. B 117 (1982) 175.

[53] W. Fischler, B. Ratra, and L. Susskind, Quantum Mechanics of Inflation, Nucl. Phys. B 259 (1985) 730, (E) 268 (1986) 747.

[54] J.M. Bardeen, P.J. Steinhardt, and M.S. Turner, Spontaneous Creation of Almost Scale-Free Density Perturbations in an Inflationary Universe, Phys. Rev. D 28 (1983) 679.

[55] V.F. Mukhanov, H.A. Feldman, and R.H. Brandenberger, Theory of cosmological perturbations. Part 1. Classical perturbations. Part 2. Quantum theory of perturbations. Part 3. Extensions, Phys. Reports 215 (1992) 203.

[56] R.K. Sachs and A.M. Wolfe, Perturbations of a cosmological model and angular variations of the microwave background, Astrophys. J. 147 (1967) 73.

[57] V.A. Rubakov, M.V. Sazhin, and A.V. Veryaskin, Graviton Creation in the Inflationary Universe and the Grand Unification Scale, Phys. Lett. B 115 (1982) 189; R. Fabbri and M.D. Pollock, The Effect of Primordially Produced Gravitons upon the Anisotropy of the Cosmological Microwave Background Radiation, Phys. Lett. B 125 (1983) 445; L.F. Abbott and M.B. Wise, Constraints on Generalized Inflationary Cosmologies, Nucl. Phys. B 244 (1984) 541; B. Allen, The Stochastic Gravity Wave Background in Inflationary Universe Models, Phys. Rev. D 37 (1988) 2078; M.J. White, Contribution of long wavelength gravitational waves to the cosmic microwave background anisotropy, Phys. Rev. D 46 (1992) 4198. 
[58] G. Lazarides, Q. Shafi, and N.D. Vlachos, Supersymmetric inflation, baryogenesis and $v_{\mu}-v_{\tau}$ oscillations, Phys. Lett. B 427 (1998) 53; G. Lazarides and N.D. Vlachos, Hierarchical neutrinos and supersymmetric inflation, Phys. Lett. B 459 (1999) 482; G. Lazarides, Supersymmetric hybrid inflation, NATO Sci. Ser. II 34 (2001) 399 [hep-ph/0011130].

[59] W. Buchmüller, P. Di Bari, and M. Plümacher, Leptogenesis for pedestrians, Annals Phys. 315 (2005) 305.

[60] C. Callan, R. Dashen, and D. Gross, The Structure of the Gauge Theory Vacuum, Phys. Lett. B 63 (1976) 334; R. Jackiw and C. Rebbi, Vacuum Periodicity in a Yang-Mills Quantum Theory, Phys. Rev. Lett. 37 (1976) 172.

[61] N.S. Manton, Topology in the Weinberg-Salam Theory, Phys. Rev. D 28 (1983) 2019; F.R. Klinkhamer and N.S. Manton, A Saddle Point Solution in the Weinberg-Salam Theory, Phys. Rev. D 30 (1984) 2212.

[62] G.'t Hooft, Symmetry Breaking Through Bell-Jackiw Anomalies, Phys. Rev. Lett. 37 (1976) 8; G.'t Hooft, Computation of the Quantum Effects Due to a Four-Dimensional Pseudoparticle, Phys. Rev. D 14 (1976) 3432.

[63] J.A. Harvey and M.S. Turner, Cosmological baryon and lepton number in the presence of electroweak fermion number violation, Phys. Rev. D 42 (1990) 3344; L.E. Ibáñez and F. Quevedo, Supersymmetry protects the primordial baryon asymmetry, Phys. Lett. B 283 (1992) 261.

[64] P.M. Garnavich et al., Supernova limits on the cosmic equation of state, Astrophys. J. 509 (1998) 74; A.G. Riess et al., Observational Evidence from Supernovae for an Accelerating Universe and a Cosmological Constant, Astron. J. 116 (1998) 1009; S. Perlmutter et al., Measurements of $\Omega$ and $\Lambda$ from 42 high redshift supernovae, Astrophys. J. 517 (1999) 565.

[65] R.R. Caldwellet, R. Dave, and P.J. Steinhardt, Cosmological imprint of an energy component with general equation of state, Phys. Rev. Lett. 80 (1998) 1582.

[66] P. Binetrui, Cosmological constant versus quintessence, Int. J. Theor. Phys. 39 (2000) 1859; E.J. Copeland, M. Sami, and S. Tsujikawa, Dynamics of dark energy, Int. J. Mod. Phys. D 15 (2006) 1936.

[67] M.E. Gomez, G. Lazarides, and C. Pallis, Supersymmetric cold dark matter with Yukawa unification, Phys. Rev. D 61 (2000) 123512; J.R. Ellis, T. Falk, K.A. Olive, and M. Srednicki, Calculations of neutralino-stau coannihilation channels and the cosmologically relevant region of MSSM parameter space, Astropart. Phys. 13 (2000) 181, (E) 15 (2001) 413.

[68] G. Belanger, F. Boudjema, A. Pukhov, and A. Semenov, micrOMEGAs 3 : A program for calculating dark matter observables, Comput. Phys. Commun. 185 (2014) 960.

[69] J. Preskill, M.B. Wise, and F. Wilczek, Cosmology of the Invisible Axion, Phys. Lett. B 120 (1983) 127; L.F. Abbott and P. Sikivie, A Cosmological Bound on the Invisible Axion, Phys. Lett. B 120 (1983) 133; M. Dine and W. Fischler, The Not So Harmless Axion, Phys. Lett. B 120 (1983) 137.

[70] M. Kawasaki, E. Sonomoto, and T.T. Yanagida, Cosmologically allowed regions for the axion decay constant $F_{a}$, Phys. Lett. B 782 (2018) 181 and references therein.

[71] G. Lazarides, C. Panagiotakopoulos, and Q. Shafi, Axion, $\mu$ term, and supersymmetric hybrid inflation, Phys. Rev. D 95 (2017) 055017.

[72] E. Aprile et al., Dark Matter Search Results from a One Ton-Year Exposure of XENON1T, Phys. Rev. Lett. 121 (2018) 111302. 
[73] J.R. Ellis, K.A. Olive, Y. Santoso, and V.C. Spanos, Gravitino dark matter in the CMSSM, Phys. Lett. B 588 (2004) 7; K. Jedamzik, K.-Y. Choi, L. Roszkowski, and R. Ruiz de Austri, Solving the cosmic lithium problems with gravitino dark matter in the CMSSM, J. Cosmol. Astropart. Phys. 07 (2006) 007; J. Pradler and F.D. Steffen, Implications of Catalyzed BBN in the CMSSM with Gravitino Dark Matter, Phys. Lett. B 666 (2008) 181.

[74] L. Covi, L. Roszkowski, R. Ruiz de Austri, and M. Small, Axino dark matter and the CMSSM, J. High Energy Phys. 06 (2004) 003; H. Baer, A.D. Box, and H. Summy, Neutralino versus axion/axino cold dark matter in the 19 parameter SUGRA model, J. High Energy Phys. 10 (2010) 023; K.-Y. Choi, L. Covi, J.E. Kim, and L. Roszkowski, Axino Cold Dark Matter Revisited, J. High Energy Phys. 04 (2012) 106.

[75] E.S. Abers and B.W. Lee, Gauge Theories, Phys. Reports 9 (1973) 1 and references therein.

[76] J.H. Schwarz, Superstring theory, Phys. Reports 89 (1982) 223.

[77] L. Susskind, The Anthropic Landscape of String Theory, arXiv:hep-th/0302219.

[78] N. Ganoulis, G. Lazarides, and Q. Shafi, Classification of Three Generation Superstring Models According to Their Discrete Symmetries, Nucl. Phys. B 323 (1989) 374.

[79] E. Witten, Five-branes and M theory on an orbifold, Nucl. Phys. B 463 (1996) 383.

[80] G. Lazarides and N.D. Vlachos, Initial conditions for supersymmetric inflation, Phys. Rev. D 56 (1997) 4562.

[81] M. Gasperini and G. Veneziano, Pre-big bang in string cosmology, Astropart. Phys. 1 (1993) 317.

[82] G. Lazarides and N. Tetradis, Two stage inflation in supergravity, Phys. Rev. D 58 (1998) 123502; C. Panagiotakopoulos and N. Tetradis, Two stage inflation as a solution to the initial condition problem of hybrid inflation, Phys. Rev. D 59 (1999) 083502.

[83] P.A.R. Ade et al., Detection of $\overline{\mathbf{B}}$-Mode Polarization at Degree Angular Scales by BICEP2, Phys. Rev. Lett. 112 (2014) 241101. 